\title{
International treatment guidelines for anaemia in chronic kidney disease - what has changed?
}

\author{
Balancing the risks and benefits of erythropoietin-stimulating agents and iron therapy
}

Robert J MacGinley MB BS, FRACP, Nephrologist and Convenor of Medicine

Rowan G Walker

MD, FRACP, MPH Director and Professor

1 Deakin Medical School Geelong, VIC.

2 Department of Renal Medicine, Alfred Hospital, Melbourne, VIC.

rob.macginley@ deakin.edu.au

doi: 10.5694/mjal3.10538 ne in nine Australians has chronic kidney disease (CKD), ${ }^{1}$ although the condition may often not be recognised in primary care. ${ }^{2}$ There are five stages of CKD, ranging from Stage 1, in which patients have normal renal function but urinary abnormalities, structural abnormalities or genetic traits pointing to kidney disease, through to Stage 5, in which patients have end-stage disease. ${ }^{1}$

Although anaemia in patients with CKD is multifactorial in origin, it is primarily associated with relative erythropoietin production deficiency ${ }^{3}$ as the glomerular filtration rate (GFR) falls. Once the estimated GFR trends below $60 \mathrm{~mL} / \mathrm{min} / 1.73 \mathrm{~m}^{2}$ (Stage 3a CKD), erythropoietin production by the kidneys falls, and anaemia may develop.

The history of anaemia management in CKD and associated clinical practice guidelines has been one of contradiction and perceived industry influence. ${ }^{4}$ The publication in August 2012 by the Kidney Disease: Improving Global Outcomes (KDIGO) group (an international collaboration of nephrologists) of a guideline that updates and informs clinical practice in this area was therefore welcome.

The KDIGO guideline contains 47 recommendations with varying grades of evidence (Box) ${ }^{5}$ It is noteworthy that the conclusions, recommendations and ungraded suggestions for clinical practice in the KDIGO guideline are largely consistent with those currently provided in the Kidney Health Australia-Caring for Australasians with Renal Impairment (KHA-CARI) guidelines. ${ }^{6,7}$ Of particular note, the KDIGO guideline takes into account the importance of balancing the risks and benefits of erythropoietin-stimulating agents (ESAs) and iron therapy.

A key aspect of the KDIGO guideline is that it recommends more caution in the use of erythropoietin. The cloning of human erythropoietin 30 years ago was an important breakthrough that led to the clinical development of recombinant human erythropoietin and thus to the use of ESAs for treating anaemia in patients with CKD. Numerous observational studies suggested that patients with lower targeted haemoglobin levels had worse outcomes than those with higher targets. ${ }^{4}$ This conclusion was supported by the clinical consequences of anaemia (including fatigue, exercise intolerance, cognitive impairment and exacerbation of cardiovascular disease $)^{3}$ and led clinicians to virtually demand ESAs for the treatment of CKD-associated anaemia. It was therefore ironic that the first randomised controlled trials to evaluate the outcomes of higher versus lower haemoglobin targets, using recombinant human erythropoietin, found the unexpected reverse outcome of negative effects (increased cardiovascular events and mortality) in patients randomly assigned to the higher targets. ${ }^{8,9}$

The evidence now seems to suggest that haemoglobin target levels between 100 and $115 \mathrm{~g} / \mathrm{L}$ should be the aim for patients with CKD, and certainly not levels $>130 \mathrm{~g} / \mathrm{L},{ }^{10}$ which have the potential to cause harm. In fact, the KDIGO guideline reaffirms the very strong (Grade 1A) KHA-CARI recommendation of not targeting haemoglobin concentrations $>130 \mathrm{~g} / \mathrm{L}$. ${ }^{7,10}$ There are also newer cautionary recommendations, not covered by the KHACARI guidelines, regarding ESA use in patients with active malignancy (1B), a history of stroke (1B) or a history of malignancy (2C), where there is greater potential for harm.

The recommendations in the KDIGO guideline relating to the use of iron supplementation are similar to those in the KHA-CARI guidelines. ${ }^{6}$ They include the recommendation that oral iron supplementation is inadequate compared with injectable iron. This is problematic for most general practitioners, as intravenous iron cannot be administered in general practice, due to the need for monitoring. However, intravenous iron therapy may sometimes be arranged for the patient at a local hospital. Further, the KDIGO guideline recommends the use of iron indices to help guide therapy, with considerations of infection risk from excess iron and suboptimal ESA responsiveness.

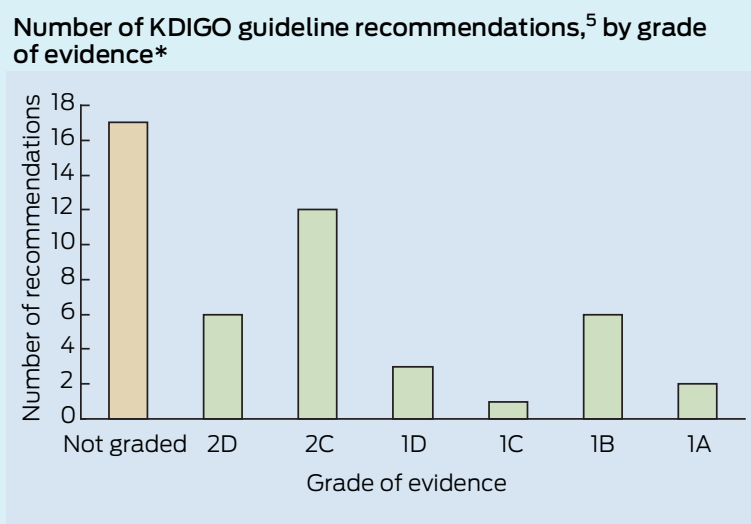

KDIGO = Kidney Disease: Improving Global Outcomes. * Using the Grading of Recommendations Assessment, Development, and Evaluation (GRADE) system. 
The KDIGO guideline also covers the use of red cell transfusion in patients with $\mathrm{CKD}^{5}$ again emphasising the importance of balancing risks and benefits. For example, for patients who may undergo future organ transplantation, red cell transfusion carries a risk of sensitisation to various tissue-type antigens; and, for all patients, excessive blood transfusions can lead to iron overload diseases. Symptom change (eg, decrease in fatigue) in an individual patient, rather than a haemoglobin concentration threshold, is a better measure of successful improvement.

Importantly, the first section of the KDIGO guideline considers the diagnosis and evaluation of anaemia in later stages of CKD (Stage $3 a$ and beyond), where anaemia is most common. ${ }^{3}$ The recommendations, while not graded, provide protocol-type approaches to the frequency of testing and a rational approach to diagnosis that is relevant and appropriate in the Australian context. As GPs frequently manage patients with Stage $3 a$ and $3 b$ CKD, when CKD-associated anaemia is most likely to develop, it is expected that the KDIGO guideline will assist them in investigating and excluding other reversible causes of anaemia in the earlier stages of CKD.

In summary, for GPs and other non-nephrologist clinicians, the key KDIGO guideline recommendations are to: (i) consider CKD in any diagnostic workup of anaemia; (ii) recognise that injectable (rather than oral) iron is the first-line treatment for CKD-associated anaemia; (iii) consider ESA use only when the haemoglobin level is about $100 \mathrm{~g} / \mathrm{L}$ - refer the patient to a nephrologist at that time; and, lastly, (iv) be appropriately cautious with the use of transfusions (blood and platelets) if transplantation potential exists.

Competing interests: No relevant disclosures.

Provenance: Commissioned; externally peer reviewed.

1 Kidney Health Australia. Key chronic kidney disease (CKD) statistics in Australia. http://www.kidney.org.au/HealthProfessionals/CKDinAustralia/ tabid/622/Default.aspx (accessed May 2013).

2 Razavian M, Heeley EL, Perkovic V, et al. Cardiovascular risk management in chronic kidney disease in general practice (the AusHEART study). Nephrol Dial Transplant 2012; 27: 1396-1402.

3 Mercadal L, Metzger M, Casadevall, et al. Timing and determinants of erythropoietin deficiency in chronic kidney disease. Clin J Am Soc Nephrol 2012; 7: 35-42.

4 Goldsmith D. 2009: a requiem for rHuEPOs - but should we nail down the coffin in 2010? Clin J Am Soc Nephrol 2010; 5: 929-935.

5 Kidney Disease: Improving Global Outcomes (KDIGO) Anemia Work Group. KDIGO clinical practice guideline for anemia in chronic kidney disease. Kidney Int Suppl 2012; 4: 279-335.

6 MacGinley R, Walker R, Irving M. KHA-CARI Guidelines. Use of iron in chronic kidney disease patients [draft]. August 2012. http://www.cari.org.au/ DIALYSIS_bht_published/DRAFT_Iron_18_March_2013.pdf (accessed Apr 2013).

7 McMahon L, MacGinley R. KHA-CARI Guidelines. Biochemical and haematological targets: haemoglobin levels in patients using erythropoiesis stimulating agents. October 2011. http://www.cari.org.au/DIALYSIS_bht_ published/KHA_CARI_Anaemia_guidelines_November_\%202011.pdf (accessed Apr 2013).

8 Besarab A, Bolton WK, Browne JK, et al. The effects of normal as compared with low hematocrit values in patients with cardiac disease who are receiving hemodialysis and epoetin. NEngl J Med 1998; 339: 584-590.

9 Pfeffer MA, Burdmann EA, Chen CY, et al; TREAT Investigators. A trial of darbepoetin alfa in type 2 diabetes and chronic kidney disease. N Engl J Med 2009; 361: 2019-2032.

10 Palmer SC, Navaneethan SD, Craig JC, et al. Meta-analysis: erythropoiesisstimulating agents in patients with chronic kidney disease. Ann Intern Med 2010; 153: 23-33. 